\title{
Hybrid Sliding-Window Based Detector for Spectrum Sensing in Radar Bands
}

\author{
Dayan Adionel Guimarães, and Chang Heon Lim
}

\begin{abstract}
The Gershgorin radii and centers ratio (GRCR) and the Gini index detector (GID) have been recently proposed as robust solutions with low computation complexity for centralized cooperative spectrum sensing. Subsequently, the sliding-window based versions of them, named SGRCR and SGID, have been devised for spectrum sensing in radar bands. While the SGID has superior performance in the specific scenario of dominant propagation path, i.e, high Rice factor $K$, the SGRCR exhibits relatively small performance variations with $K$, but is outperformed by the SGID when $K$ is high. In this paper, motivated by the complementary behaviors of the GRCR and the GID, a hybrid sliding-window based GRCR-GID (HSGG) detection strategy is proposed. The new test statistic is formed by the weighted sum of the GRCR and GID test statistics for each sliding sensing window. Numerical results demonstrate that the hybrid detector combines the best attributes of the base detectors, achieving high performances under any Rice factor. Specifically, it performs a little worse than the SGRCR and much better than the SGID when $K=0$, and as good as the SGID for mild-to-high values of $K$. Moreover, there are cases in which the HSGG can beat the SGRCR and the SGID.
\end{abstract}

Index Terms-Cognitive radio, dynamic spectrum access, GID, GRCR, HSGG, SGID, SGRCR, radar, spectrum sensing.

\section{INTRODUCTION}

D URING the last few years, we are witnessing an unprecedented growth of telecommunication services, especially with regard to wireless communication systems. As a consequence, the radio-frequency spectrum has become an increasingly congested or even scarce resource in certain bands, mainly due to the traditional static spectral allocation policy, which does not take into account the variability of utilization of some spectrum assigned to the primary networks. The problem tends to become more serious with the massification of the Internet of Things (IoT) and the deployment of the fifth generation $(5 \mathrm{G})$ of wireless communication networks.

The concept of cognitive radio (CR) has emerged as a promising solution to the above problem [1]. It can implement a dynamic spectrum allocation policy in which idle frequency bands can be opportunistically occupied by secondary user (SU) terminals.

D. A. Guimarães is with the National Institute of Telecommunications (Instituto Nacional de Telecomunicações - Inatel), Santa Rita do Sapucaí, MG, Brazil. E-mail: dayan@inatel.br

C. H. Lim is with the Department of Electronic Engineering, Pukyong National University, Busan, Republic of Korea. E-mail: chlim@pknu.ac.kr.

This work was supported in part by CNPq, Grant 308365/2017-8, and RNP, with resources from MCTIC, Grant 01250.075413/2018-04, under the Radiocommunication Reference Center (Centro de Referência em Radiocomunicações, CRR) project of the National Institute of Telecommunications (Instituto Nacional de Telecomunicações, Inatel), Brazil. doi: 10.14209/jcis.2019.20.
For the successful access to a vacant frequency band, cognitive SU terminals must be able to detect the presence of the primary user (PU) signal in the band of interest, by means of a process known as spectrum sensing [2], [3].

Spectrum sensing can be realized independently by each cognitive SU, or it can be cooperative. In the latter case, the accuracy of the decisions upon the occupancy of the sensed band may be improved compared to the non-cooperative sensing, owed to the spatial diversity produced by SUs separated in space in a fading channel environment.

In centralized cooperative spectrum sensing with data fusion, which is the object of the present work, a soft decision or hard decision from a received signal at each SU are conveyed to a fusion center (FC), where the global decision is made. This decision is subsequently informed to the SUs, allowing them to access the band if it is found to be vacant, through some multiple access technique. The transmissions of the spectrum sensing data from the SUs to the FC, and the broadcast transmission of the global decision from the FC to the SUs are made via dedicated or shared control channels.

Recently, it has been realized that radar bands are potential candidates for cognitive secondary networks, since these bands are wide and are considerably underutilized [4]. In consonance with this fact, the Federal Communications Commission (FCC) has already regulated wireless local area network (WLAN) devices to detect radar signals [5] in the $5 \mathrm{GHz}$ band. Thus, the successful operation of these devices in radar bands requires the spectrum sensing functionality.

A pulse radar refers to a radar system that applies short duration radio-frequency (RF) pulses as probe signals. Typically, a sequence of such pulses carrying high energy are radiated periodically and sparsely in time, meaning that a pulse radar signal has a low duty-cycle. Motivated by these characteristics, the authors of [6] devised an spectrum sensing technique in which the conventional single detection event carried out during a sensing interval is replaced by multiple short-time intermediate detections made in a sliding-window fashion, thus exploiting the sparsity of the signal to be detected. After the sliding-window reaches the end of the sensing interval, the multiple intermediate sensing results are combined to yield the global decision upon the occupation of the sensed band.

The single specific requirement for the choice of the detector to be adopted in the sliding-window approach is a low computational complexity. This is because the multiple intermediate detections must be accomplished during the regular sensing interval, which is small compared to the communication interval [7]. In [6], the Gerschgorin radii and centers ratio (GRCR) detector proposed in [8] has been chosen. Another 
detector exhibiting an implementation complexity very close to the GRCR is the Gini index detector (GID) [9], which makes it also attractive to the sliding-window approach. The GRCR and the GID share the following important attributes:

1) They are completely blind, i.e., they do not demand knowledge of any characteristic of the signal to be detected, and do not use the noise variance information in their test statistics;

2) They carry one of the lowest computational complexities known so far, overcame only by the well-known energy detector (which is semi-blind due to the need of the noise variance information);

3) They exhibit the constant false alarm rate (CFAR) property, which means that the false alarm rate is maintained at a target level, no matter the noise variance;

4) They are robust, meaning that their performances are mildly affected in the scenario of noise and received signal powers that may not be the same over the SUs receivers, and may vary over time.

In [6], the application of the GRCR to the sliding-window spectrum sensing approach coined the term sliding GRCR (SGRCR). Similarly, the application of the GID to this approach has been named sliding GID (SGID) in [10].

It has been found in [10] that the SGID can outperform the SGRCR in the majority of system parameterizations and scenarios, but its robustness is somewhat affected when the radar signal duty-cycle is high, and its performance is penalized in the absence of dominant path in the sensing channel (this last flaw is inherited from the GID). The performance of the sliding-window based energy detector (SED) has also been assessed in [10], serving as a benchmark for quantifying the performances of the SGRCR and the SGID.

In this paper, the SGRCR and the SGID are combined, via a weighted $\operatorname{sum}^{1}$, into a single test statistic. The resultant detector is named hybrid sliding-window based GRCRGID (HSGG). It is demonstrated that the HSGG achieves a combination of the merits of the SGRCR and the SGID, being capable of overcoming these base detectors in some system paramerizations and scenarios. It is emphasized that the HSGG attains improved performances in the absence of dominant propagation path with respect to the SGID, and has its robustness restored when the radar duty-cycle is high.

The remainder of this paper is organized as follows. Section II describes the system model, and the GRCR and the GID detectors. Section III is devoted to the proposed HSGG detector. A large set of numerical results are presented in Section IV. Section V concludes the paper and gives some directions for further related research.

\section{SYSTEM MODEL}

It is known that the adherence of system simulation outcomes to the ones obtained from real-life experiments strongly depends on the accuracy and completeness of the system model. Likewise in [6], [10], here it is adopted a very close-toreal simulation model that considers the sensing channel (the

\footnotetext{
${ }^{1}$ The weighted sum of test statistics has been also investigated in [11], not in the context of the sliding-window based radar signal detection problem.
}

one between the radar transmitter to the spectrum sensors) as a combination of an additive white Gaussian noise (AWGN) channel with multipath fading, also taking into account different degrees of dominance of signal propagation paths or, equivalently, different levels of line-of-sight $(\mathrm{LoS})$. Moreover, considering that the SUs' terminals are mobile devices, and that the SUs' front-ends are not perfectly equal to one another and may be subjected to different environmental temperatures, the thermal noise powers affecting the SUs will be different with probability one, as well as time-varying. Yet, owing to the fact that the SUs' positions are mobile and random, it is expected that the received signal powers across the SUs will not be uniform (equal to one another), and will vary over time. These unequal and time-varying quantities are hereafter referred to as nonuniform-dynamical noise and signal powers.

\section{A. Signal model}

A cooperative spectrum sensing (CSS) scheme is considered, with $m$ cognitive SUs collecting $m n$ samples ( $n$ samples per SU) of the radar signal received during a given sensing interval. At the fusion center (FC) of the secondary cognitive network, the received signal matrix $\mathbf{Y} \in \mathbb{C}^{m \times n}$ is given by

$$
\mathbf{Y}=\mathbf{h} \mathbf{x}^{\mathrm{T}}+\mathbf{V}
$$

where the superscript $\mathrm{T}$ denotes transposition, and $\mathbf{h}=\left[h_{1}, h_{2}, \ldots, h_{m}\right]^{\mathrm{T}}$ is the channel vector with $h_{i}$ representing the complex channel gains between the radar transmitter and the $i$-th SU receiver, for $i=1, \ldots, m$. These gains are assumed to be constant during the sensing interval, and independent and identically distributed (i.i.d.) between consecutive sensing rounds. The assumption of constant channel gain means that the sensing interval is smaller than the coherence time of the fading channel. The independence comes from the assumption that the time between two consecutive sensing rounds is larger than the coherence time of the channel. The equal distribution for the fading in all sensing rounds means that the fading statistics do not change over time, i.e. the fading process is stationary. Moreover, the multiplicative channel model in Equation (1) subsumes a frequency flat fading channel, meaning that the sensing channel delay spread is small and, as a consequence, the signal bandwidth is smaller than the channel coherence bandwidth.

The channel vector in Equation (1) is modeled as $\mathbf{h}=\mathbf{G a}$ where the diagonal gain matrix $\mathbf{G} \in \mathbb{R}^{m \times m}$ is given by $\mathbf{G}=\operatorname{diag}\left(\sqrt{\mathbf{p} / p_{\text {avg }}}\right)$, with $\mathbf{p}=\left[p_{1}, p_{2}, \ldots, p_{m}\right]^{\mathrm{T}}$ being the vector with the received signal power levels in each SU, where $p_{\text {avg }}=\frac{1}{m} \sum_{i=1}^{m} p_{i}$ is the average received signal power over all SUs. If the overall channel power gain is unitary (without loss of generality), the radar signal is transmitted

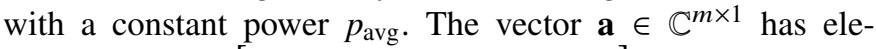
ments $a_{i} \sim \mathbb{C} \mathcal{N}[\sqrt{K /[2(K+1)]}, 1 /(K+1)]$ for $i=1, \ldots, m$, guaranteeing unitary second moment of the fading magnitude, where $K$ is the Rice factor ${ }^{2}$.

\footnotetext{
${ }^{2}$ In a multipath Ricean fading channel, the Rice factor is the ratio between the dominant and the remaining path powers. A larger $K$ means a stronger line-of-sight (LoS) received signal. If $K=0$, the Ricean fading specializes to the Rayleigh fading, which corresponds to no LoS.
} 
The matrix $\mathbf{V} \in \mathbb{C}^{m \times n}$ in Equation (1) contains independent zero mean complex Gaussian noise samples. To consider the possibility of nonuniform noise variances across the SUs' receivers, the elements in the $i$-th row of $\mathbf{V}$ are assumed to have variance $\sigma_{i}^{2}, i=1, \ldots, m$.

The vector $\mathbf{x} \in \mathbb{R}^{n \times 1}$ in Equation (1) represents the samples of the radar signal. In practice, a pulse radar signal is formed by a series of short-time pulses with duration of about 1-5 microseconds [5]. The time between the start of consecutive pulses is typically on the order of 1 millisecond, yielding a low duty-cycle waveform. From the perspective of the detector, the received signal from a rotating radar is seen as a series of bursts of pulses. The number of pulses in a burst depends on the aperture of the antennas and on the speed of the radar antenna rotation. The time from the start of one burst to the start of the next one is typically on the order of 1-10 seconds. Hence, in the lowpass equivalent representation, the radar signal vector can be expressed by $\mathbf{x}=[\ldots, v, v, \ldots, v, 0,0, \ldots, 0, v, v, \ldots, v, 0,0, \ldots, 0, \ldots]^{\mathrm{T}}$, where the amplitude $v$ is set according to the desired radar signal power $p_{\text {avg }}$ and duty-cycle $D$, i.e., $v=\sqrt{p_{\text {avg }} / D}$. The number of consecutive $v$ s (i.e., the pulse width $W_{\mathrm{p}}$ expressed in samples) and consecutive 0s (i.e., the pulse spacing, also expressed in samples) are set to yield the desired duty-cycle.

The number of pulses within a sensing interval is assumed to be a uniform random variable $\mathcal{U} \sim[1, N]$ to represent the asynchronous operation between the radar bursts and the spectrum sensing interval, where $N$ is the number of pulses within a burst. Due to this synchronousness, the approximate received signal-to-noise ratio ( $\mathrm{SNR}$ ), in $\mathrm{dB}$, averaged over all SUs, is given by [6]

$$
\mathrm{SNR} \approx 10 \log _{10}\left[\frac{(N+1) p_{\text {avg }}}{2 N \sigma_{\text {avg }}^{2}}\right],
$$

where $\sigma_{\text {avg }}^{2}=\frac{1}{m} \sum_{i=1}^{m} \sigma_{i}^{2}$ is the average noise variance. This is approximation because the number of radar pulses seen by the SUs during a sensing interval may be a non integer, and the expression assumes that this number is an integer, for simplicity.

\section{B. GRCR and GID test statistics}

In the conventional (non sliding-window based) cooperative spectrum sensing, the FC computes the received signal sample covariance matrix $(\mathrm{SCM})$ as

$$
\mathbf{R}=\frac{1}{n} \mathbf{Y} \mathbf{Y}^{\dagger},
$$

where $\dagger$ denotes the complex conjugate and transpose.

The GRCR test statistic [8] is calculated according to

$$
T_{\mathrm{GRCR}}=\frac{\sum_{i=1}^{m} \sum_{j=1, j \neq i}^{m}\left|r_{i j}\right|}{\sum_{i=1}^{m} r_{i i}},
$$

where $r_{i j}$ is the element in the $i$-th row and $j$-th column of $\mathbf{R}$, for $i, j=1, \ldots, m$.

The GID test statistic [9], apart from a constant factor that does not influence performance, is given by

$$
T_{\mathrm{GID}}=\frac{\sum_{i=1}^{m^{2}}\left|r_{i}\right|}{\sum_{i=1}^{m^{2}} \sum_{j=1}^{m^{2}}\left|r_{i}-r_{j}\right|},
$$

where $r_{i}$ is the $i$-th element of the vector $\mathbf{r}$ formed by stacking all columns of $\mathbf{R}$.

\section{Performance metrics}

The metrics often used to assess the spectrum sensing performance are the probability of detection and the probability of false alarm, respectively denoted as $P_{\mathrm{d}}=\operatorname{Pr}\left(\right.$ decision $\left.=\mathcal{H}_{1} \mid \mathcal{H}_{1}\right)$ and $P_{\text {fa }}=\operatorname{Pr}\left(\right.$ decision $\left.=\mathcal{H}_{1} \mid \mathcal{H}_{0}\right)$, where $\mathcal{H}_{1}$ and $\mathcal{H}_{0}$ are the hypotheses of the presence (i.e. $\mathbf{Y}=\mathbf{h} \mathbf{x}^{\mathrm{T}}+\mathbf{V}$ ) and absence (i.e. $\mathbf{Y}=\mathbf{V}$ ) of the radar signal, respectively, and $\operatorname{Pr}(E)$ is the probability of the underlying event $E$. A higher value of $P_{\mathrm{d}}$ is desirable to reduce the interference caused by the secondary network to the primary network due to missed detections. On the other hand, $P_{\text {fa }}$ needs to be small for more opportunistic transmissions by the secondary network.

A typical tool for analyzing these metrics simultaneously is the receiver operating characteristic (ROC) curve, which trades $P_{\mathrm{fa}}$ versus $P_{\mathrm{d}}$ as the decision threshold of the corresponding detector is varied. A condensed metric also often used is the area under the ROC curve, which is called the AUC. The worst detection performance, which corresponds to a ROC curve with $P_{\mathrm{d}}=P_{\mathrm{fa}}$, gives $\mathrm{AUC}=0.5$. The possibly best detection performance corresponds to a ROC curve attaining $P_{\mathrm{d}}=1$ and $P_{\mathrm{fa}}=0$, yielding $\mathrm{AUC}=1$. Hence, we can say that the AUC of a general detector has a range over between 0.5 and 1.

\section{SLIDING-WINDOW BASED HYBRID GRCR-GID}

\section{A. The HSGG test statistic}

The sliding-window detection technique works by successively shifting a small sensing window through the whole sensing interval [6]. An intermediate decision on the presence or absence of the radar signal is made for each step of the sliding-window. When the window reaches the end of the sensing interval, a logic operation is made among the intermediate decisions to yield the final global decision.

The technique works under the divide-and-conquer principle: it exchanges the reduced performance of individual smaller sensing windows by an increase of the captured energy of a radar pulse that by chance falls into one or more intermediate sensing windows. Moreover, the noise energy during such small sensing windows is smaller than the one present in the whole sensing interval, potentially resulting in performance improvements of the radar signal detection.

Figure 1 illustrates the operation of the sliding-window based spectrum sensing approach, assuming some hypothetical quantities [6]. In this figure, a single radar signal burst with $N$ pulses (3 in this example) during the sensing interval is shown.

The beginning of the first pulse is a random variable, since, as already mentioned, the radar burst and the sensing interval are not synchronous to each other. Each pulse lasts an interval corresponding to $W_{\mathrm{p}}$ samples (20 in this example). The slidingwindow has a size of $S_{\mathrm{w}}$ samples and moves through the sensing interval (600 samples in this example) in $S_{\mathrm{n}}$ steps of size $S_{\mathrm{s}}$ samples. The number of steps is the total number 


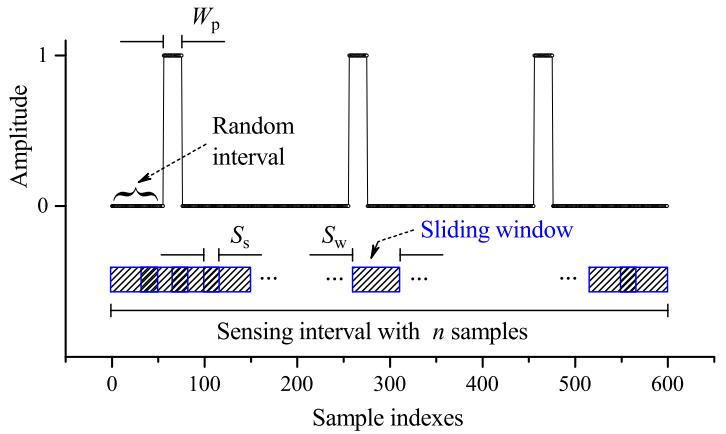

Fig. 1. Radar burst with $N=3$ pulses, pulse width $W_{\mathrm{p}}=20$ samples, and duty-cycle $D=10 \%$. The step size is $S_{\mathrm{s}}$, and the window size is $S_{\mathrm{w}}$. The whole sensing interval corresponds to $n$ samples. CCIEEE [6].

of intermediate sensing rounds made during the entire sensing interval. It is given by [6]

$$
S_{\mathrm{n}}=\left(n-S_{\mathrm{w}}\right) / S_{\mathrm{s}}+1 .
$$

Moreover, if $n$ samples are collected by each SU during the sensing interval, from Figure 1 the radar signal duty-cycle is easily found to be $D=N W_{\mathrm{p}} / n$, yielding

$$
n=W_{\mathrm{p}} N / D \text {. }
$$

In each intermediate sensing round number $k$, with $k=1, \ldots, S_{\mathrm{n}}$, the proposed HSGG test statistic is formed from the weighted sum of $T_{\mathrm{GRCR}}$ and $T_{\mathrm{GID}}$, that is,

$$
T_{\mathrm{HSGG}}(k)=T_{\mathrm{GRCR}}(k)+w f(m) T_{\mathrm{GID}}(k),
$$

where $T_{\mathrm{GRCR}}(k)$ and $T_{\mathrm{GID}}(k)$ respectively denote the GRCR and GID test statistics for the $k$-th intermediate sensing round, and $w f(m)$ is the overall weighting function with an adjustable weight parameter $0 \leq w \leq 1$.

Given the test statistics $T_{\mathrm{HSGG}}(k)$ computed in the $k$-th intermediate sensing round, the global decision at the FC is made after the OR-logic operation among them. This is equivalent to say that the decision is made in favor of the presence of the radar signal if $\max \left\{T_{\mathrm{HSGG}}(1), T_{\mathrm{HSGG}}(2), \ldots, T_{\mathrm{HSGG}}\left(S_{\mathrm{n}}\right)\right\}>\gamma_{\mathrm{HSGG}}$, where $\gamma_{\mathrm{HSGG}}$ is the decision threshold for the HSGG detector.

When the SGRCR or the SGID is implemented alone, a similar procedure applies, that is, given the test statistics $T_{\mathrm{GRCR}}(k)$ or $T_{\mathrm{GID}}(k)$ computed in the $k$-th intermediate sensing round according to (4) and (5), the global decision at the $\mathrm{FC}$ is made in favor of the presence of the radar signal if $\max \left\{T_{\mathrm{GRCR}}(1), T_{\mathrm{GRCR}}(2), \ldots, T_{\mathrm{GRCR}}\left(S_{\mathrm{n}}\right)\right\}>\gamma_{\mathrm{GRCR}}$, or $\max \left\{T_{\mathrm{GID}}(1), T_{\mathrm{GID}}(2), \ldots, T_{\mathrm{GID}}\left(S_{\mathrm{n}}\right)\right\}>\gamma_{\mathrm{GID}}$, where $\gamma_{\mathrm{GRCR}}$ and $\gamma_{\mathrm{GID}}$ are the decision thresholds for the GRCR and GID detectors, respectively.

It can be seen in (4) and (5) that $T_{\mathrm{GRCR}}$ and $T_{\mathrm{GID}}$ depend explicitly on $m$, but also depend implicitly on other system parameters that influence the entries of $\mathbf{R}$, for example the SNR, the number of samples and the sensing channel Rice factor. Nonetheless, it has been found empirically that the dependence of $T_{\mathrm{GRCR}}$ and $T_{\mathrm{GID}}$ on $m$ dominates the other ones, and this is the reason for defining the weighting function $f(m)$ as a function of $m$ solely. The role of this function is to guarantee that different values of $m$ do not change the chosen value of the weight $w$; otherwise, a new $w$ would have to be determined for each $m$. The performance results in Section IV will confirm that this is an adequate simplification. The next subsection is devoted on determining $w f(m)$.

\section{B. Determining the weighting function $w f(m)$}

If a closed-form expression for some performance metric of the proposed HSGG detector were available, the optimal weighting function $w f(m)$ could be determined analytically or numerically. Since the parametric probability density functions (PDFs) of $T_{\mathrm{GRCR}}$ and $T_{\mathrm{GID}}$ are unknown, the PDF of $T_{\mathrm{HSGG}}$ cannot be determined analytically. Thus, it is not possible to find the optimum $w f(m)$ analytically. Even though all involved PDFs were known, the optimum $w f(m)$ would not be a single value for a given $w$ and $m$, since the weighted sum (8) implicitly calls for a trade-off between the performances of the base test statistics, and, as already mentioned, the actual weighting function does not depend solely on $m$.

The empirical procedure for determining $f(m)$ was accomplished as follows:

1) Some spectrum sensing performance results in terms of AUC, obtained from computer simulations, were generated for several representative values of $m$. Since the performance changes with $m$, different values of SNR were chosen to yield the best AUC close to 0.9 (which represents a satisfactory performance) for all pairs of $m$ and SNR;

2) For each pair of $m$ and SNR, the sub-optimum overall weight $w f(m)$ was determined as the one beyond which the AUC produced by the HSGG is little affected (almost saturated) in the situation of strong path dominance ( $K=10$, i.e. almost pure-AWGN channel). The results are presented in Figure 2, where the AUCs for no path dominance (Rice factor $K=0$, i.e. Rayleigh fading channel) are also given. For example, notice that the AUCs of the HSGG for $K=10$ do not improve significantly beyond $w f(m)=18$ for $m=4$ and beyond $w f(m)=700$ for $m=8$, staying very close to the AUCs of the SGID. Additionally, notice that, at these values of $w f(m)$, the corresponding AUCs of the HSGG for $K=0$ have decreased by less than half of its maximum difference from that of the SGRCR. Following this procedure, other values of $m$ and SNR where analyzed, yielding the sub-optimum $w f(m)$ values listed in Table I;

3) The pairs $m$ and $w f(m)$ were then applied to a leastsquares $^{3}$ power curve fit to the model function $\mathrm{am}^{b}$, yielding $a \approx 0.025$ and $b \approx 4.84$. The coefficient of determination [13] related to the curve fit result was found to be $R^{2} \approx 0.997$, meaning that the model function accurately describes the data given in Table I. Thus, taking into account that the adjustable weight parameter $w$ conve-

\footnotetext{
${ }^{3}$ The power fit can be made via any nonlinear curve fitting toll, adopting any error fit minimization method. As a simple alternative, it can be made via the command line power fit $\{\{3,3\},\{4,18\},\{5,50\},\{6$, $120\},\{7,300\},\{8,700\},\{9,1000\},\{10,1800\}\}$ in the WolframAlpha public website [12], which is based on the software Mathematica.
} 

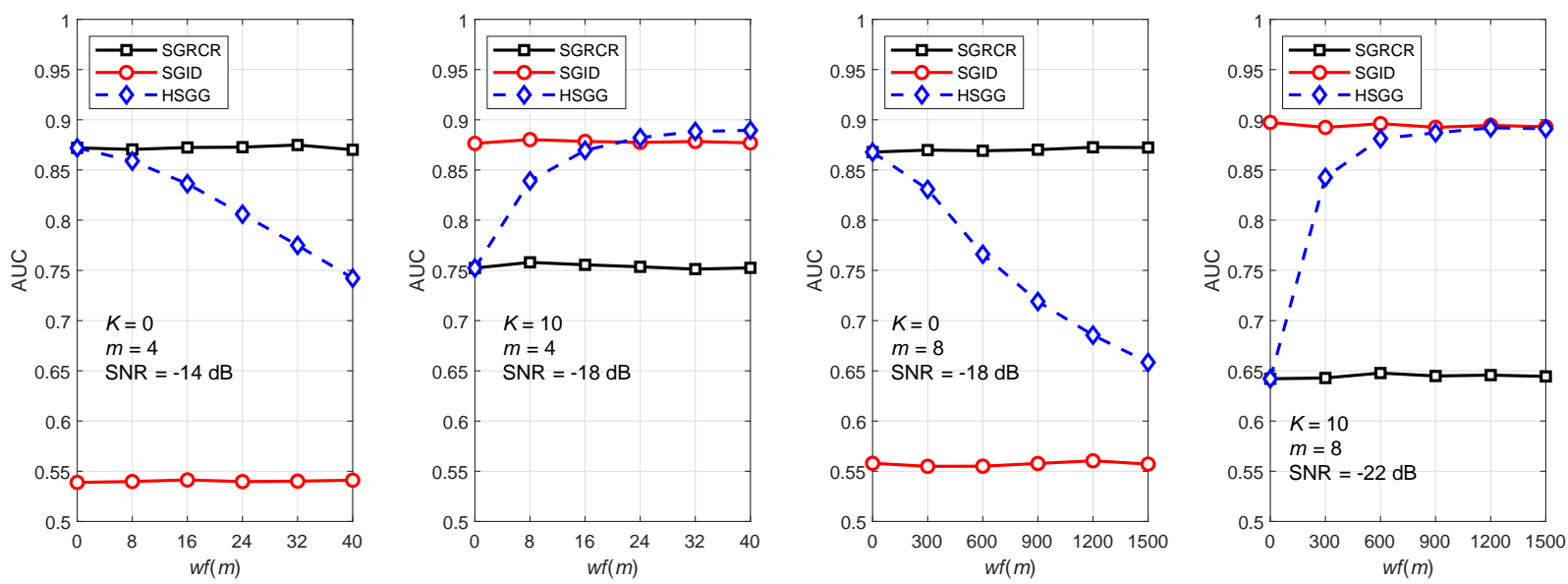

Fig. 2. AUCs versus $w f(m)$ for $n=1200, D=0.05, W_{\mathrm{p}}=S_{\mathrm{s}}=S_{\mathrm{w}}=15, N=4, S_{\mathrm{n}}=80$, nonuniform-dynamical signal and noise: $K=0, m=4$ and SNR $=-14 \mathrm{~dB}$ (left); $K=10, m=4$ and SNR $=-18 \mathrm{~dB}$ (middle-left); $K=0, m=8$ and SNR $=-18 \mathrm{~dB}$ (middle-right); $K=10, m=8$ and $\mathrm{SNR}=-22 \mathrm{~dB}$ (right).

TABLE I

SUB-OPTIMUM VALUES OF $w f(m)$ FOR DIFFERENT PAIRS OF $m$ AND SNR, ASSUMING $K=10, n=1200, D=0.05, W_{\mathrm{P}}=S_{\mathrm{S}}=S_{\mathrm{W}}=15$, $N=4, S_{\mathrm{N}}=80$, NONUNIFORM-DYNAMICAL SIGNAL AND NOISE.

\begin{tabular}{ccc}
\hline$m$ & SNR, dB & $w f(m)$ \\
\hline 3 & -17 & 3 \\
4 & -18 & 18 \\
5 & -19 & 50 \\
6 & -20 & 120 \\
7 & -21 & 300 \\
8 & -22 & 700 \\
9 & -23 & 1000 \\
10 & -24 & 1800 \\
\hline
\end{tabular}

niently lies in the interval $[0,1]$ by definition, an appropriate overall weight function is

$$
w f(m)=w 0.05 m^{4.84} .
$$

4) The adjustable weight that yields a good balance between the performance loss with respect to the SGRCR when $K=0$, and the proximity to the SGID when $K=10$ is $w=0.3$, which corresponds one third of the ranges of $w f(m)$ shown in Figure 2.

5) Finally, the proposed HSGG test statistics that is applied hereafter in the $k$-th intermediate sensing round, for any $w$, becomes

$$
T_{\mathrm{HSGG}}(k)=T_{\mathrm{GRCR}}(k)+w 0.05 m^{4.84} T_{\mathrm{GID}}(k) .
$$

\section{Computational complexity}

As reported in [8], [9], the computational complexity of the GRCR is $O\left(\mathrm{~nm}^{2}\right)$. This complexity is owed to the fact that the computation of each element of the matrix $\mathbf{R}$ requires $n$ complex multiplications plus $(n-1)$ complex additions over the elements of $\mathbf{Y}$. Since a complex multiplication requires 4 real multiplications plus 2 real additions, it follows that the computation of $\mathbf{R}$ requires a total of $4 \mathrm{~nm}^{2}$ real multiplications plus $2 m^{2}(2 n-1)$ real additions. Thus, the computational complexity in the big- $O$ notation can be determined by the computational time growth rate, which is dominated by $\mathrm{nm}^{2}$.

The complexity $O\left(\mathrm{~nm}^{2}\right)$ is $m$ times larger than the complexity of the well-known energy detector, which is the smallest one (one must recall that the energy detector is not fully blind). Hence, taking into account that the number $m$ of SUs in cooperation is not large in practice, the complexity of the GRCR shall not be much larger than that of the energy detector. The GID has roughly the same complexity of the GRCR, also due to the burden of computing the matrix $\mathbf{R}$. Hence, the GRCR and the GID are the least complex blind detectors available in the literature, making them attractive to the sliding-window approach.

Regarding the sliding-window technique, the additional complexity of making the weighted sum of $T_{\mathrm{GRCR}}(k)$ and $T_{\mathrm{GID}}(k)$ to form $T_{\mathrm{HSGG}}(k)$ is small compared to the complexity of computing the SCM that is used to calculate $T_{\mathrm{GRCR}}(k)$ and $T_{\mathrm{GID}}(k)$. Moreover, as demonstrated in [6], the computational complexity for obtaining $S_{\mathrm{n}} \mathrm{SCMs}$ in terms of complex multiplications is $S_{\mathrm{n}} S_{\mathrm{w}} m^{2}$, which turns out to be $n m^{2}$ when $S_{\mathrm{w}}=S_{\mathrm{s}}$; see (6). Thus, when $S_{\mathrm{w}}=S_{\mathrm{s}}$ the computational complexity of the HSGG does not vary with the sliding window size $S_{\mathrm{w}}$.

It is worth emphasizing that the sliding-window approach has its parameters fully configurable to allow for a trade-off between the spectrum sensing performance and the overall sensing speed. For instance, if the window size $S_{\mathrm{w}}$ fits the pulse width $W_{\mathrm{p}}$, the maximum radar pulse energy can be captured, improving the sensing performance. If the step size is $S_{\mathrm{s}}=1$, the chance of hitting a radar pulse is maximized at the expense of a large number of intermediate sensing rounds, yielding a large processing time [6].

\section{Performance Results}

In this section we present results in terms of AUCs, as determined by different values of the main system parameters that are relevant to the spectrum sensing performance. These 
results were produced by computer simulations using the MATLAB version R2018a, from 30000 Monte Carlo events in which the received signal matrix $\mathbf{Y}$ was generated under the hypothesis $\mathcal{H}_{1}$ (to estimate $P_{\mathrm{d}}$ ) and $\mathcal{H}_{0}$ (to estimate $\left.P_{\text {fa }}\right)$. The AUCs were computed using the MATLAB function -trapz $\left(P_{\mathrm{fa}}, P_{\mathrm{d}}\right)$, from ROC curves containing 200 pairs of $\left(P_{\mathrm{fa}}, P_{\mathrm{d}}\right)$. The MATLAB source code is available in [14].

When a given parameter is not the one that is varied, its value was set to: $m=4$ SUs; radar signal with duty-cycle $D=5 \%$; maximum of $N=4$ pulses per burst during the sensing interval under the $\mathcal{H}_{1}$ hypothesis; $n=1200$ samples collected by each $\mathrm{SU} ; W_{\mathrm{p}}=15$ samples per radar pulse; sensing window size $S_{\mathrm{w}}$ equal to $W_{\mathrm{p}}$ and equal to the step size $S_{\mathrm{s}}$ of the sliding-window; average $\mathrm{SNR}=-15 \mathrm{~dB}$; sensing channel Rice factor $K=10$; and weight factor $w=0.3$ in Equation (10).

When noise and signal powers are uniform (i.e., the same across the SUs and constant over time), $\sigma_{i}^{2}=\sigma_{\text {avg }}^{2}=1$, and $p_{i}=p_{\text {avg }}$ according to the desired SNR; see (2). In the more realistic scenario in which noise and signal powers are nonuniform-dynamical (i.e., different across the SUs and timevarying), $\sigma_{i}^{2}$ and $p_{i}$ are respectively assumed to have a uniform distribution over $\left[0.05 \sigma_{\mathrm{avg}}^{2}, 1.95 \sigma_{\mathrm{avg}}^{2}\right]$ and $\left[0.05 p_{\mathrm{avg}}, 1.95 p_{\mathrm{avg}}\right]$ in each spectrum sensing event.

The beginning of the first radar pulse with respect to the beginning of the sensing interval is supposed to be uniformly distributed over $\left[0, n-W_{\mathrm{p}}-1\right]$. By adopting such random delay, it is guaranteed that at least a single entire pulse is present during a whole sensing interval, under $\mathcal{H}_{1}$.

Figure 3 gives AUCs versus the sliding window size $S_{\mathrm{w}}$, assuming uniform (U) and nonuniform-dynamical (NUD) noise and received signal powers for two values of the Rice factor, $K=0$ and $K=10$. Since $W_{\mathrm{p}}=15, N=4$ and $D=0.05$, it follows from (7) that $n=1200$ samples. Additionally, given that $S_{\mathrm{w}}=S_{\mathrm{s}}=15,30,60,120,240,600$, and 1200 samples, from (6) it follows that $S_{\mathrm{n}}=80,40,20,10,5,2$, and 1 intermediate sensing rounds per sensing interval, respectively. The following can be concluded from Figure 3:

1) The detection of a radar signal using the SGRCR and the HSGG benefits from the sliding-window approach for any $K$, meaning that the spectrum sensing performance can be improved for small window sizes (recall that a conventional non-sliding-window based detection results if $S_{\mathrm{w}}=n$ );

2) If $K=0$, the SGID does not benefit from the slidingwindow approach at all. In general, as $S_{\mathrm{w}}$ decreases, the SNR for a sensing round gets higher and the GRCR detector benefits from it. However, the enhancement of the SNR does not mean to decrease the variation of the elements of an SCM and, as a consequence, the performance of the SGID does not improve as $S_{\mathrm{w}}$ is reduced;

3) The SGRCR is little affected by the nonuniform-dynamical noise and received signal powers, i.e., its robustness has been inherited from the GRCR detector proposed in [8];

4) The SGID inherited the robustness of the GID proposed in [9] only for very small window sizes;

5) The proposed HSGG is roughly as robust as the GRCR for any window size;
6) In the case of small window sizes, which is the target in the sliding-window technique, the performance of the HSGG is far above the SGID when $K=0$, and is close to the SGID when $K=10$. The HSGG performs closely to the SGRCR when $K=0$, beating this detector when $K=10$.

Since the robustness of the HSGG has been illustrated by Figure 3, hereafter only the more practical-appealing nonuniform-dynamical noise and received signal power is considered.

The AUCs versus the sensing channel Rice factor $K$ are presented in Figure 4 for some different values of SNR. The following conclusions can be drawn from this figure:

1) All detectors improve their performances as $K$ increases, which is an expected result, but in different amounts;

2) All graphs in this figure make it evident that the performance of the SGID gets worse than the SGRCR and the HSGG when $K$ is close to zero. Nonetheless, its performance gets better rapidly with increasing $K$, which stems from the fact that higher $K$ enhances the GID test statistic for the case of $\mathcal{H}_{1}$ [9];

3) The GRCR and GID hybridization proved to yield the desired outcome: the HSGG attains approximately the same performance of the SGRCR when $K=0$, significantly outperforming the SGID in this situation. Moreover, the HSGG performs closely to the SGID when $K$ is large, producing better performance than the SGRCR at smaller SNRs.

Figures 5 and 6 depict AUCs versus the average SNR across the SUs, and versus the number $m$ of SUs, respectively. We can obtain the following conclusions from these figures:

1) As expected, larger values of SNR or $m$ yield better spectrum sensing performances for all detectors, but in a diminishing-return fashion and in different amounts;

2) The HSGG and the SGRCR have approximately the same performances for all SNRs and $m$ when $K$ is small, significantly outperforming the SGID;

3) As expected, the SGID recovers its attractive performance for large values of $K$, independent of the values of SNR and $m$.

4) The HSGG closely follows the SGID when $K$ is large, being capable of beating the SGRCR;

5) The relatively low value of $K=1$ is enough for the HSGG to outperform the SGRCR for any SNR and $m$, which remains the case if $K$ is increased further;

6) At larger SNRs, the HSGG is even capable of outperforming the SGID, as also noticed from Figure 4.

The AUCs as functions of the radar signal duty-cycle $D$, and of the radar pulse width $W_{\mathrm{p}}$ are shown in Figures 7 and 8 , respectively. The following conclusions apply:

1) In the case of Figure 7 , since $W_{\mathrm{p}}=15, N=4$, and $D=$ $0.01,0.02,0.05,0.1,0.2$, it follows from (7) that $n=6000$, $3000,1200,600$, and 300, respectively. Similarly, applying $S_{\mathrm{w}}=S_{\mathrm{s}}=W_{\mathrm{p}}=15$ in Equation (6) results in $S_{\mathrm{n}}=400$, 200, 80, 40, and 20, respectively; 

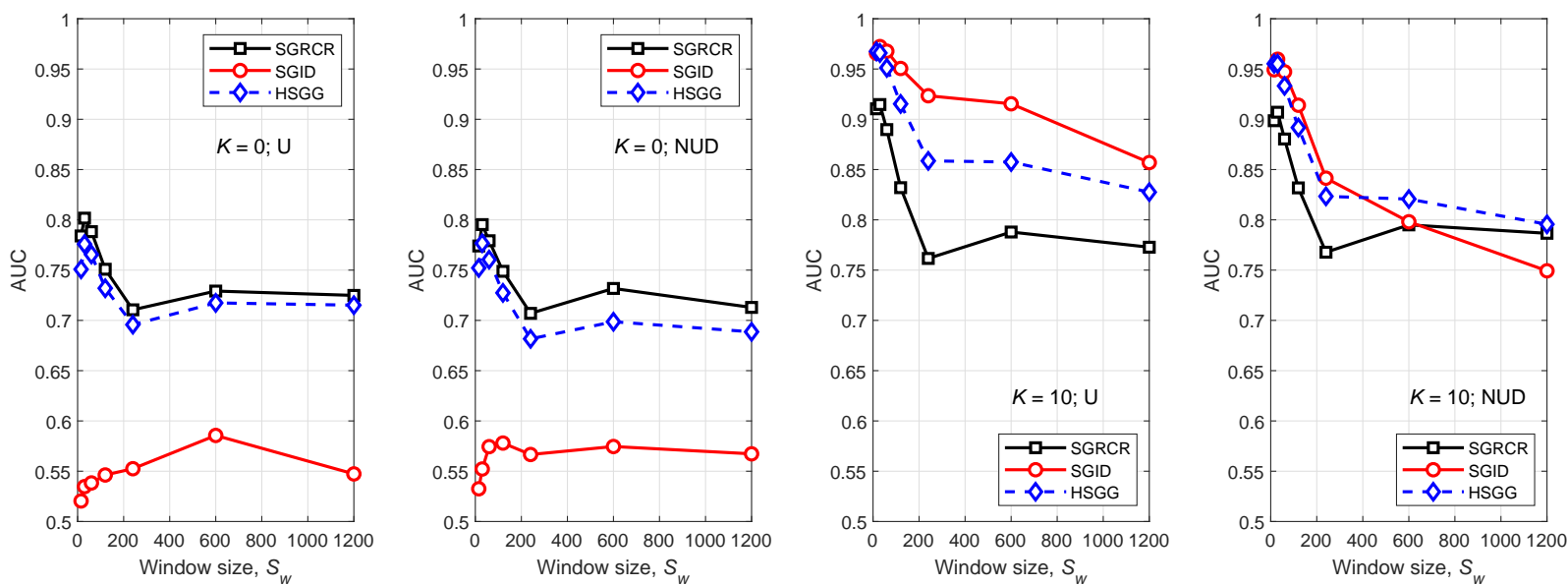

Fig. 3. AUCs versus window size $\left(S_{\mathrm{w}}\right)$ for $m=4, n=1200, D=0.05, W_{\mathrm{p}}=15, S_{\mathrm{s}}=S_{\mathrm{w}}, N=4, w=0.3$, and SNR $=-16 \mathrm{~dB}: K=0, \mathrm{U}$ (left); $K=0$, NUD (middle-left); $K=10$, U (middle-right); $K=10$, NUD (right), where U (resp. NUD) denote uniform (resp. nonuniform-dynamical) signal and noise.
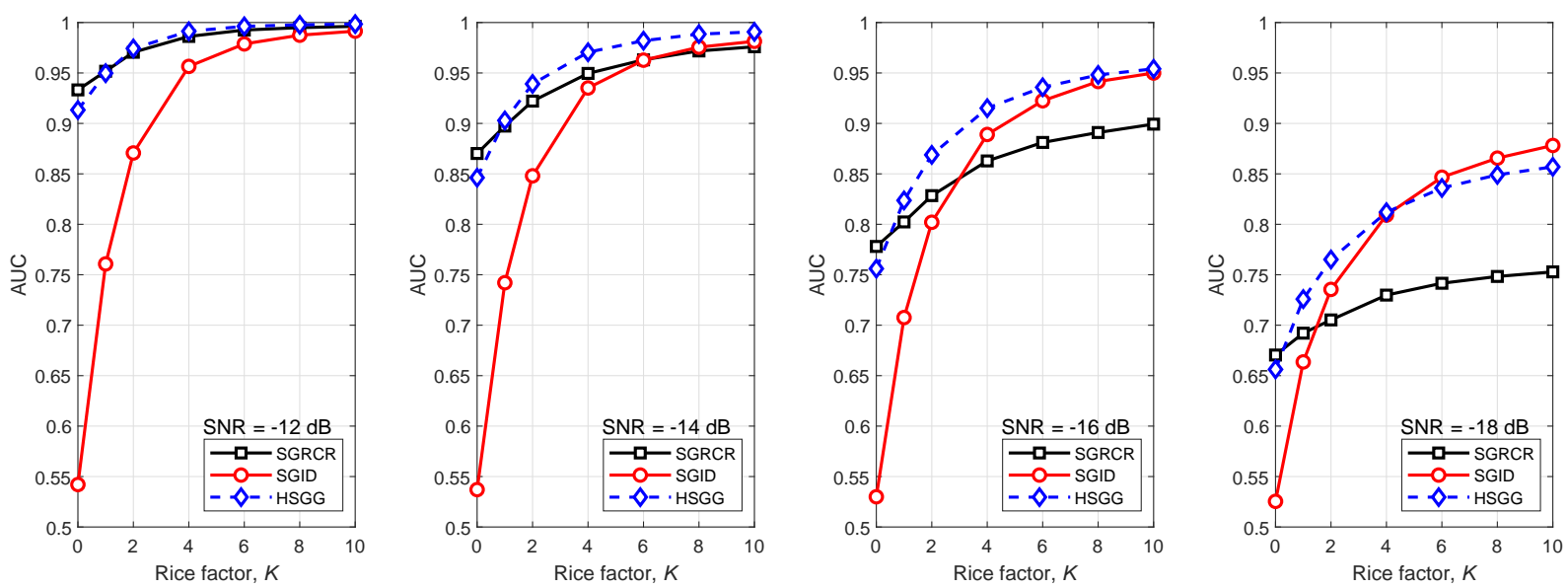

Fig. 4. AUCs versus $K$ for $m=4, n=1200, D=0.05, W_{\mathrm{p}}=S_{\mathrm{s}}=S_{\mathrm{w}}=15, N=4, S_{\mathrm{n}}=80, w=0.3$, nonuniform-dynamical signal and noise: $\mathrm{SNR}=-12 \mathrm{~dB}$ (left); SNR $=-14 \mathrm{~dB}$ (middle-left); SNR $=-16 \mathrm{~dB}$ (middle-right); $\mathrm{SNR}=-18 \mathrm{~dB}$ (right).
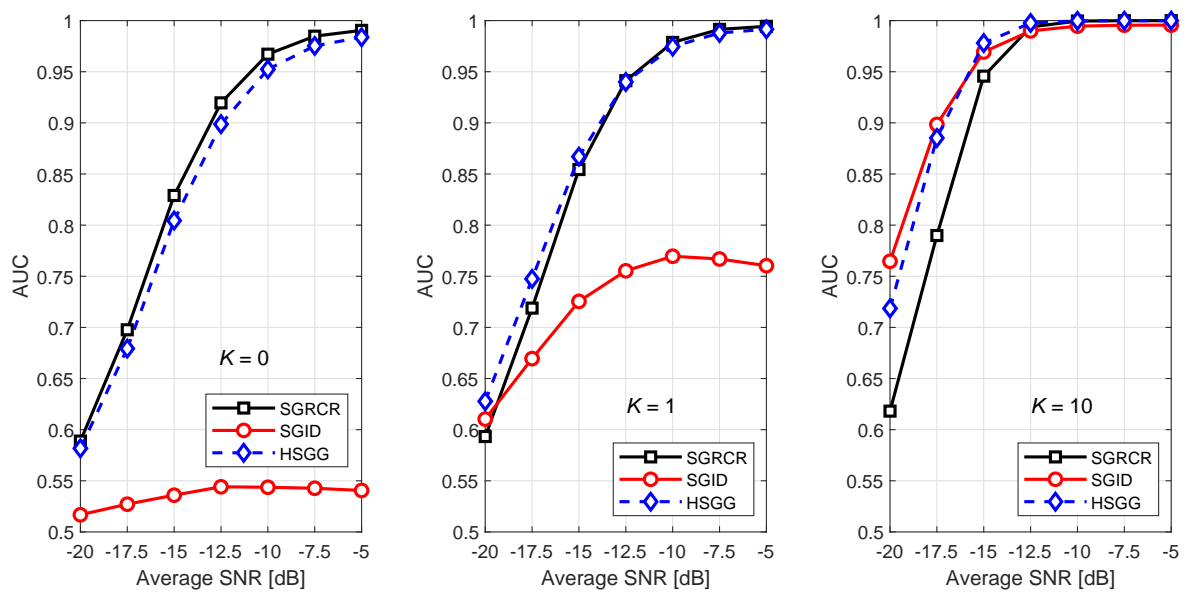

Fig. 5. AUCs versus average SNR for nonuniform noise and signal powers, $m=4, n=1200, D=0.05, W_{\mathrm{p}}=S_{\mathrm{s}}=S_{\mathrm{w}}=15, w=0.3$, and $N=4: K=0$ (left); $K=1$ (middle); $K=10$ (right). 

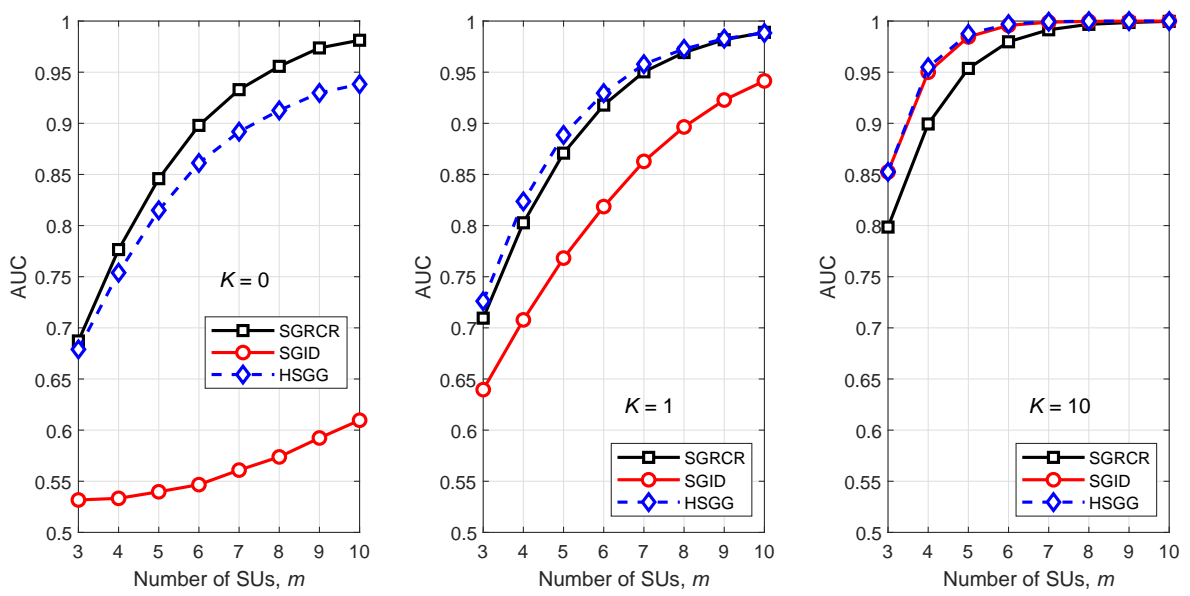

Fig. 6. AUCs versus the number of SUs $(m)$ for nonuniform noise and signal powers, $\mathrm{SNR}=-16 \mathrm{~dB}, n=1200, D=0.05, W_{\mathrm{p}}=S_{\mathrm{S}}=S_{\mathrm{w}}=15, w=0.3$, and $N=4: K=0$ (left); $K=1$ (middle); $K=10$ (right).
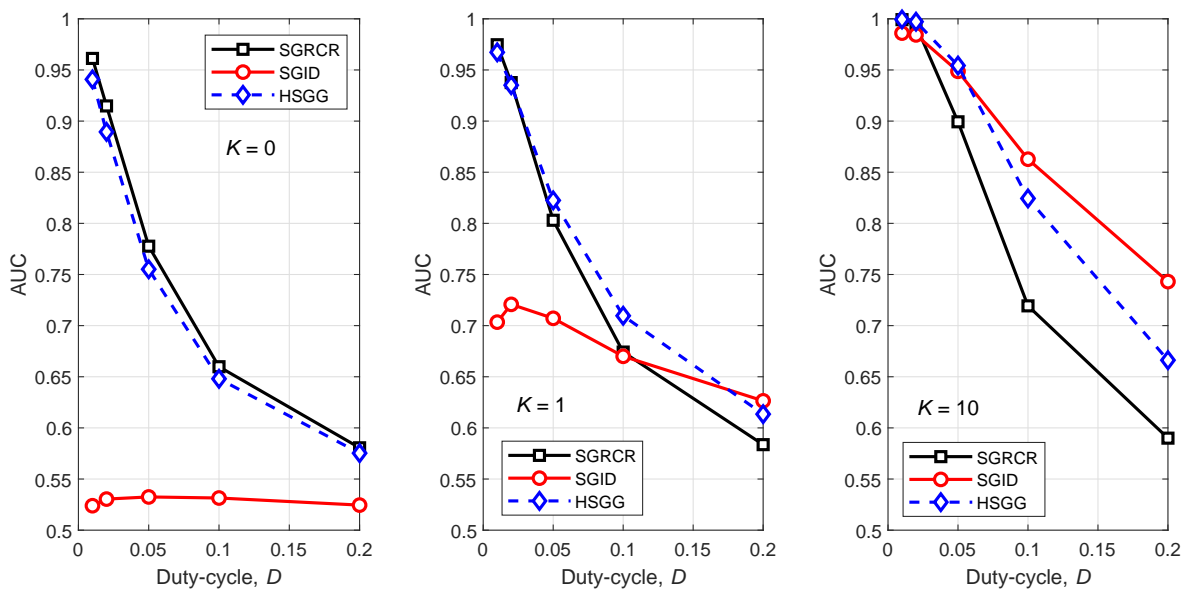

Fig. 7. AUCs versus the radar signal duty-cycle $(D)$ for nonuniform noise and signal powers, $m=4, \mathrm{SNR}=-16 \mathrm{~dB}, W_{\mathrm{p}}=S_{\mathrm{S}}=S_{\mathrm{W}}=15, w=0.3$, and $N=4: K=0$ (left); $K=1$ (middle); $K=10$ (right).
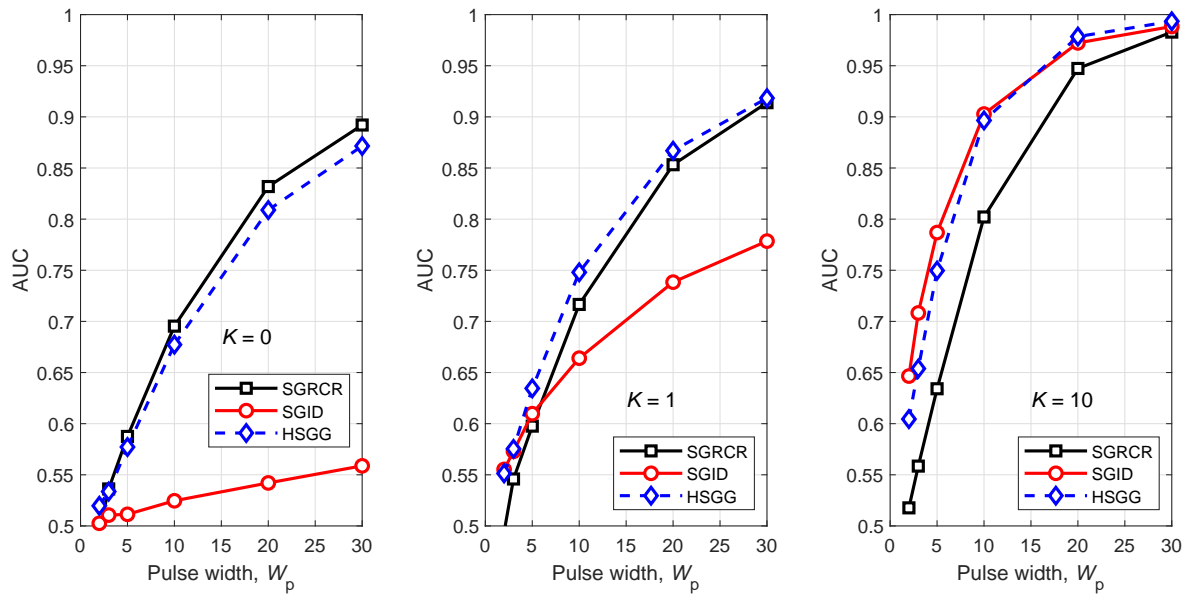

Fig. 8. AUCs versus the radar pulse width $\left(W_{\mathrm{p}}\right)$ for nonuniform noise and signal powers, $D=0.05, m=4$, SNR $=-16 \mathrm{~dB}, W_{\mathrm{p}}=S_{\mathrm{s}}=S_{\mathrm{w}}, w=0.3$, and $N=4: K=0$ (left); $K=1$ (middle); $K=10$ (right) 
2) In the case of Figure 8 , since $W_{\mathrm{p}}=S_{\mathrm{s}}=S_{\mathrm{w}}=2,3,5$, 10,20 , and $30, N=4, D=0.05$, and $S_{\mathrm{n}}=80$, it follows from (7) that $n=160,240,400,800,1600$ and 2400;

3) From the above two items, it can be seen that the variation of $D$ and $W_{\mathrm{p}}$ are accompanied by the variation of other performance-relevant parameter, the number of samples $n$, as determined from (7) and (6). Thus, Figure 7 and Figure 8 must be interpreted in terms of relative performance measures instead of absolute ones.

4) The SGID consistently remains with poor performances when $K=0$, achieving results comparable with the SGRCR and the HSGG only at higher values of $K$;

5) With a mild dominant propagation path, represented by $K=$ 1, the performance difference between the HSGG and the SGRGR increases softly as $D$ increases, which can be also seen at higher values of $K$.

6) The performance of the SGRCR at different values of $D$ practically does not vary significantly with $K$. The relatively smaller dependency of the SGRCR on $K$ has been also observed in Figure 4.

7) Recalling that a typical pulse radar signal has a small dutycycle, Figure 7 indicates that the hybrid detector performs as almost good as the SGRCR when $K=0$, exhibiting a little higher performance than the SGID when $K$ is high;

8) The difference between the performances of the HSGG and the SGRCR practically does not depend on $W_{\mathrm{p}}$, but increases as $K$ increases beyond 1 .

\section{Conclusions}

In this paper we proposed the hybrid sliding-window based GRCR-GID (HSGG) test statistic, which employs the weighted sum of the GRCR and GID test statistics in each intermediate sensing round of the sliding-window technique for its test statistic. Through a large set of numerical results, we have demonstrated that the hybrid detector combines the good attributes of the base detectors, achieving high performances in the Ricean fading channel with any level of the Rice factor. In the majority of situations and scenarios, the performance of the HSGG is slightly worse than that of the SGRCR and by far better than that of the SGID when $K=0$, and very close to the SGID for mild-to-higher values of $K$, specifically just above $K=1$. In a few cases, the HSGG demonstrated its performance better than both the SGRCR and the SGID. The comprehensive comparisons of the HSGG with the two base detectors can serve as references to parameterize the HSGG detector, when seeking for system configurations that can extract the maximum of its capabilities.

As opportunities for future related research, other combinations of the GRCR and GID test statistics, or even of the intermediate decisions from both detectors could be investigated. The sensing channel model could be also extended to include shadowing and frequency selectivity. Specifically regarding the latter one, it is conjectured that the slidingwindow based detector could exploit the multipath propagation over a frequency-selective fading channel, if most of the received pulse replicas could be resolved by the channel and could be efficiently caught by the sliding window, similarly to what happens in the rake receiver in the context of directsequence spread spectrum communications.

\section{REFERENCES}

[1] J. Mitola III and G. Q. Maguire Jr., "Cognitive radio: making software radios more personal," IEEE Personal Commun. Mag., vol. 6, no. 4, pp. 13-18, Aug. 1999, doi: 10.1109/98.788210.

[2] I. F. Akyildiz, B. F. Lo, and R. Balakrishnan, "Cooperative spectrum sensing in cognitive radio networks: A survey," $E l$ sevier Physical Comm., vol. 4, pp. 40-62, Mar. 2011, doi: 10.1016/j.phycom.2010.12.003.

[3] Y. Arjoune and N. Kaabouch, "A comprehensive survey on spectrum sensing in cognitive radio networks: Recent advances, new challenges, and future research directions," Sensors, vol. 19, no. 1, 2019, doi: $10.3390 / \mathrm{s} 19010126$.

[4] F. Paisana, N. Marchetti, and L. A. DaSilva, "Radar, TV and cellular bands: Which spectrum access techniques for which bands?" IEEE Communications Surveys Tutorials, vol. 16, no. 3, pp. 1193-1220, April 2014, doi: 10.1109/SURV.2014.031914.00078.

[5] C. H. S. Tsai and S. Yin, "Method and device for robust signal detection in wireless communications," USA Patent US20080031386A1, Feb, 2008. [Online]. Available: https://www.google.com.br/patents/ US20080031386.

[6] D. A. Guimarães and C. H. Lim, "Sliding-window-based detection for spectrum sensing in radar bands," IEEE Communications Letters, vol. 22, no. 7, pp. 1418-1421, July 2018, doi: 10.1109/LCOMM.2018.2834932.

[7] The Institute of Electrical and Electronic Engineers, IEEE. (2011) IEEE 802 Part 22: Cognitive Wireless RAN Medium Access Control (MAC) and Physical Layer (PHY) Specifications: Policies and Procedures for Operation in the TV Bands. [Online]. Available: http://standards.ieee.org/getieee802/download/802.22-2011.pdf

[8] D. A. Guimarães, "Robust test statistic for cooperative spectrum sensing based on the Gerschgorin circle theorem," IEEE Access, vol. 6, pp. 2445-2456, 2018, doi: 10.1109/ACCESS.2017.2783443.

[9] D. A. Guimarães, "Gini index inspired robust detector for spectrum sensing over Ricean channels," Electronics Letters, November 2018, doi: 10.1049/el.2018.7375

[10] D. A. Guimarães and C. H. Lim, "Performance of simple and fast sliding window detectors for spectrum sensing in radar bands," in XXXVII Brazilian Telecommunications Symposium, SBrT'19, Sep 2019.

[11] D. A. Guimarães, "Linear combination of test statistics for cooperative spectrum sensing (original title in portuguese: combinação linear de estatísticas de teste para sensoriamento espectral cooperativo)," in XXXVII Brazilian Telecommunications Symposium, SBrT'19, Sep 2019.

[12] E. W. Weisstein. WolframAlpha public website. Visited on 04/06/2019. [Online]. Available: https://www.wolframalpha.com/

[13] G. Hahn, "The coefficient of determination exposed!" Chemical Technology, vol. 3, no. 10, pp. 609-614, Oct. 1973.

[14] D. A. Guimarães, "Compute capsule for simulating the hybrid slidingwindow-based GRCR-GID detector," https://www.codeocean.com/, June 2019, doi: https://doi.org/10.24433/CO.7965200.v1.

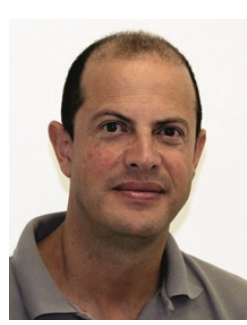

Dayan Adionel Guimarães received the $\mathrm{Ph} . \mathrm{D}$. degree in Electrical Engineering from the State University of Campinas, Unicamp, Brazil, in 2003. He is currently a Senior Lecturer and a Researcher with the National Institute of Telecommunications, Inatel, Brazil. His research interests are fixed and mobile wireless communications, radio propagation, simulation of digital communication systems, spectrum sensing for cognitive radio, and convex optimization and signal processing applied to communications.

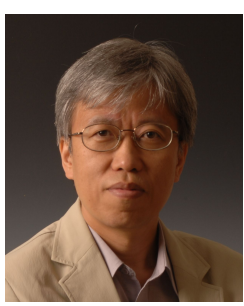

Chang Heon Lim received the Ph.D. degree in Electrical Engineering from the Korea Advanced Institute of Science and Technology (KAIST) in 1993. $\mathrm{He}$ is currently a professor with Pukyong National University, Busan, Republic of Korea. His research interests are adaptive transmission, cognitive radio, coexistence for wireless systems, radar detection, and target tracking. 